\title{
A Normative Multi-Agent Systems Approach to the Use of Conviviality for Digital Cities
}

\author{
Patrice Caire \\ University of Luxembourg, Computer Science Department \\ L-1359, Luxembourg, 6, Rue Richard Coudenhove-Kalergi, Luxembourg \\ patrice.caire@uni.lu
}

\begin{abstract}
Conviviality is a mechanism to reinforce social cohesion and a tool to reduce mis-coordination between individuals, groups and institutions in web communities, for example in digital cities. We use a two-fold definition of conviviality as a condition for social interactions and an instrument for the internal regulation of social systems. In this paper we discuss the use of normative multi-agent systems to analyze the use of conviviality for digital cities, by contrasting norms for conviviality with legal and institutional norms in digital cities. We show the role of the distinction among various kinds of norms, the explicit representation of norms, the violability of norms, the dynamics of norms and the creation of norms in the context of conviviality.
\end{abstract}

Keywords. Conviviality, multi-agent systems, normative systems, social computing, digital cities.

\section{Introduction}

The role of norms for conviviality is a condition for social interactions and an instrument for the internal regulation of social systems [1. For example, in digital cities "government regulations extend laws with specific guidance to corporate and public actions" [2].

In this paper we raise the following question: how can normative multi-agent systems be used to model conviviality? We approach this question focussing on conviviality in digital cities, and by contrasting the use of normative multi-agent systems for conviviality with legal and institutional norms in digital cities.

Our main question breaks down into the following research questions: What are digital cities, what are normative multi-agent systems, what is conviviality and finally, can norms be applied to conviviality?

The layout of this paper follows these sub-questions. In section 2 we give a brief overview on digital cities, in section 3 we explain norms in regards to the legal and institutional aspects of digital cities, in section 4 we present a literature survey on the notion of conviviality and in section 5 we examine the use of norms for conviviality.

Dagstuhl Seminar Proceedings 07122

Normative Multi-agent Systems

http://drops.dagstuhl.de/opus/volltexte/2007/906 


\section{Brief Overview on Digital Cities}

There are many ways to define digital cities. "They can be seen as a local social information infrastructure, providing information over the real city to locals and of course to visitors of the real city. The digital city can also be approached as a communication medium, influencing the personal networks of inhabitants of a digital neighborhood. Another view is the digital city as a tool to improve local democracy and participation, in fact the basic idea behind the digital city in Amsterdam. Fourth, we can characterize the digital city as a free space to experience and experiment with cyberspace. Finally, the digital city can be seen as a practical resource for the organization of every day life. One can think of local electronic commerce, and the provision of online public services as a support of local economic activities. However, the digital city may also become an experiment with new forms of solving problems and coordinating social life. Where currently most activities are coordinated by the market or by the state, the digital city may become a tool that enables people to do things by mobilizing the available local resources, using existing and emerging social networks" [3].

\subsection{Digital Cities Categories and Goals}

There are five Broad Categories of digital cities: Non-profit grass-roots community initiatives such as Amsterdam DDS-De Digitale Stad, municipal information and communication networks such as E-governments, commercial city oriented web sites, for instance AOL Digital Cities and Microsoft CitySearch, virtual environment for virtual communities ahd communities of interest such as the scientific community and finally social ICT experiments, often in less privileged neighborhoods.

The main goals of digital cities are to create public space, exchange social information, explore vertical markets and innovate with next generation networks. Today their principal objective is to transform and modernize the local administration to improve the level and quality of life of the population at both individual and community levels.

\subsection{American Community Networks}

Digital cities started in the US with american community networks, inspired by a tradition of community-centered, grass-roots engagements that emphasized freedom of speech and activism. Their original goal was to create a virtual information space at first non-geographically bound for example the WELL, "Whole Earth 'Lectronic Link", but that subsequently evolved into geographically bound for example Blacksburg' Electronic Village. Their main challenges were first, the lack of synergy between community networks, private companies and administrations and second, the competition between profit and non-profit organizations.

Case study 1: Blacksburg Electronic Village was built in 91 as a consortium lead by universities, among which Virginia Tech University, by regional companies such as Bell Atlantic and local authorities. It was a high profile project 
but with very little community involvement to the vision. It was constructed from a technological point of view and the first project of the kind with web interface. It rapidly grew until 95 then its activity decreased due to fundamental disagreement between all the partners' expectations. The companies looked for revenues elsewhere and universities stopped providing internet to non-university members. It is still active today but with very local focus on community use of technology and learning.

Case study 2: Seattle Community Network emerged in 92 as part of the "Computer Professionals for Social Responsibilities" group's civil activities. It was first hosted on a donated Intel 386 running a donated copy of BSDI UNIX operating system, using FreePort (Cleveland FreeNet text based) user interface software. It was lead by citizens and grew in size by cooperating with regional libraries and offering free network access and services to all: email, homepages etc. Due to continuous financial problems and competition with commercial portals the activity decreased to its current reduced level and provides free public-access network. Interestingly, among the more recent grass-roots activities is the emergence of the Seattle Community Wireless network that creates a broadband wireless metropolitan area network.

\subsection{European Digital Cities}

The goal of European digital cities is to integrate and coordinate private, public and voluntary sectors towards better regional and local information system. The European Community first launched the Telecities program in 93 later evolved with large scope programs and projects such as Eurocities, Intelcities and eAgora. For the European Community, the goal is to share ideas and technologies between all the cities to strengthen the European partnership. For cities, the goal is to use information and communication technologies to resolve social, economic and regional development issues and improve the quality of social services. Their characteristics today are to be networks generated within and for specific regions, to form complex communities based on collaborations between citizens, universities, city administrations and private companies, and to emphasize social inclusion. Their main challenge is the difficulty to integrate grass-roots communities and commercial point of views which appears in the relatively slow commercialization of services and information.

Case study 1: Amsterdam Digital City, DDS, started in 94 as a grass-roots initiative and evolved into a non-profit organization with government support and the participation of private companies. The goals of Digital Amsterdam were to support community activities and local economy, encourage political discourse by linking citizens to the administration and innovate. Its very successful interface of squares and cafes and interactive public debates inspired many other digital cities, among others digital Bristol. The issues that caused its downfall were persistent technical problems and the initial lack of common understanding and vision between the stakeholders. Digital Amsterdam exploited all the early Internet possibilities such as USENET, IRC, GOPHER, MUDs, MOOs, Telnets and Free-nets. 
Case study 2: Virtual Helsinki started in 95 as a powerful consortium of Telecom (Nokia, Elisa), the city of Helsinki, private companies such as IBM and local universities but did not include any grass-roots community nor voluntary services. It had three goals: Technological advances with for instance the use of ISDN and Video on Demand (95), DSL, Ethernet, ADSL (97), IP based Video conferencing (98), ISDN video telephony, 3-D mapping of Helsinki (99). Digital Helsinki has been highly profitable and socially relevant with citizens' participation and contribution to social cohesion. Its projects of using avatars for citizens inspired the Habbo community.

Case study 3: Bologna 'Iperbole' and Issy-les-Moulineaux. Started in 94, Bologna's great innovation was to create an open space for citizen groups to publish information and engage in debates with public officials while Issy-lesMoulineaux in France, started in 96, developped a very successful one-stop administration with online live interaction of citizens in town meetings, interactive map, and so on.

\subsection{Asian City Informatization}

Asian digital cities, actually called city informatization, emerged as government initiatives. Their goal is to develop their country through technological innovation. Singapore initiated the process in 92 and launched in 96, Japan in 94, Korea 95 and Malaysia 96 . There were attempts to integrate grass-roots activities and university driven projects in 99 with Digital Kyoto and Shanghai but the greatest challenge still remains their top-down approach based on administration activity.

\subsection{Commercial Portals}

Commercial portals started as local portals run by private companies, such as phone or web companies and airlines, competing with each other. Nowadays, global companies such as AOL and Microsoft offer city guides with services: shopping, entertainment, some local information and maps. Their general trend is to provide easy to find and search information, good maintenance of systems and frequent updates. They are effective in Asia, where they complement government agencies, but limited in scope by their top-down controlled and selected content, lack of two-way interaction with users and main purpose, e.g. advertising.

\subsection{Digital Cities Models and Future Growth}

As yet, no one model has been identified. In the US for-profit businesses and nonprofit organizations co-exist and compete, in EU the attempts are to coordinate administrations, companies and citizens while Asia pursues government directed growth. Ultimately, digital cities need to deal with the same complexity as real cities to attract and retain usage, and to function as entities that augment their physical counterparts. 
The main goals of digital cities consist to help close the digital divide be it geographic, with access everywhere, or social, with access for all, accelerate economic development, and make cities' governments more efficient and accessible. The means used by digital cities to achieve these goals are: Pluralism and participation, combined multi-disciplinary approaches, synergy between their three constitutive elements: administration, companies and citizens and finally a shared vision between all stakeholders.

The success factors of digital cities consist in achieving across institutions and communities participation, in the balance between top-down direction needed for technical infrastructure and grass-roots initiatives necessary to insure citizens' cohesion and finally in the balance between economic and civic motivations.

\section{Legal and institutional norms in digital cities}

In digital cities "government regulations extend laws with specific guidance to corporate and public actions" [2]. This legal aspect of norms is what we are concerned here as our context is digital cities, we will therefore only look at legal norms. In their introduction to normative multi-agent systems, Boella et al. give the following definition:

A normative multi-agent system is a multi-agent system together with normative systems in which agents on the one hand can decide whether to follow the explicitly represented norms, and on the other the normative systems specify how and in which extent the agents can modify the norms." 4]

We first discuss the distinction among various kinds of norms, and then we discuss four issues in this definition, illustrated by examples in digital cities.

\subsection{Kinds of norms}

Several kinds of norms are usually distinguished in normative systems. Within the structure of normative multi-agent systems Boella et al distinguish "between regulative norms that describe obligations, prohibitions and permissions, and constitutive norms that regulate the creation of institutional facts as well as the modification of the normative system itself" [5]. A third kind of norms, procedural norms, can also be distinguished "procedural norms have long been considered a major component of political systems, particularly democratic systems" states Lawrence who further define procedural norms as "rules governing the way in which political decisions are made; they are not concerned with the content of any decision except one which alters decision-making procedures" 6].

Constitutive norms: Boella et al. note several aspects of constitutive norms, one is as intermediate concept exemplified by " $\mathrm{X}$ counts as a presiding official in a wedding ceremony", "this bit of paper counts as a five euro bill" and "this piece 
of land counts as somebodys private property" [7. Searle further explains that "the institutions of marriage, money, and promising are like the institutions of baseball and chess in that they are systems of such constitutive rules or conventions" 8. In digital cities, examples are the marriage norms and voting int he sense that going through the procedure counts as a vote.

Boella et al further believe that "the role of constitutive rules is not limited to the creation of an activity and the construction of new abstract categories. Constitutive norms specify both the behavior of a system and the evolution of the system..." 5. The dynamics of normative systems is here emphasized as in norms revision, certain actions count as adding new norms for instance amendments: "the normative system must specify how the normative system itself can be changed by introducing new regulative norms and new institutional categories, and specify by whom the changes can be done" [5]. Today "US government agencies are required to invite public comment on proposed rules" [2] This is done through the digital government interface and allow revisions to be traced.

Another aspect of constitutive norms is organizational and structural, that is, how roles define power and responsibilities and how various hierarchies structure groups and individuals. "Not only new norms are introduced by the agents playing a legislative role, but also that ordinary agents create new obligations, prohibitions and permissions concerning specific agents" [5].

Regulative Norms: As stated by Boella et al., "regulative norms are not categorical, but conditional: they specify all their applicability conditions" [5] furthermore "legal systems are often modelled using regulative norms, like obligations and permissions. However, a large part of the legal code does not contain prohibitions and permissions, but definitions for classifying the commonsense world under legal categories, like contract, money, property, marriage. Regulative norms can refer to this legal classification of reality" [7. Regulative norms express constraints, obligations and prohibition for example in the Luxembourg e-city, citizens have to use pdf files and cannot use postscript formats to access the administration documents. Regulative norms also express permission, rights and powers, for example access rights or voting right if you are resident for more than 5 years or born in the city for Luxembourg. Another example is for creating a online library account on the Paris internet site, a parents' authorization is necessary if you are under 18 years old.

Procedural norms: Lawrence distinguishes two kinds of procedural norms "objective procedural norms are rules which describe how decisions are actually made in political systems; A systems objective procedural norms are a primary determinant of the content of political decisions in that they specify who actually makes decisions, who can try to influence decision makers, what political resources are legitimate and how resources may be used. Subjective procedural norms, on the other hand, are attitudes about the way in which decisions should be made" 6. Procedural norms are instrumental for individuals working in a system. In digital cities, examples are back office procedures. 


\subsection{Explicit representation}

The first property of norms in the definition of normative multi-agent systems is that norms are explicitly represented.

Norms are often given as requirements of computer systems and only implicitly represented. An example of implicit representation is a form in which you would be asked to state wether or not you keep a pet at home without mentioning to you the purpose of the information e.g. that if your answer is affirmative, you would be requested to pay a license fee or the fee would be directly taken from your bank account. Implicit representations are opaque to users and prevent governments to fulfill the democratic promise that transparency and explicit representations deliver. As users' needs for explanation and understanding of rules and regulations grow, representations have to become more explicit and personalized to their expectations. Similarly, governments'interests also reside in explicit representation to be addressed with the development of knowledge representation and reasoning mechanisms.

Current efforts are somewhat in-between implicit and explicit representation with tools for text representation and retrieval with more advanced ontologies, semantic links and search capabilities. "Businesses spend a lot of time complying with laws and regulations and worrying about what they don't know. According to a report by the Small Business Paperwork Relief Task Force, the Office of Management and Budget estimated that in fiscal year 2003, it took businesses and citizens approximately 8.2 billion hours and 320 billion dollars filling out paperwork and complying with government regulations" http://business.gov/. Indeed, this US governmental Business portal was launched in 2006 to help small businesses comply with Federal regulations, a need that was not being met by any other Federal government program.

In NYC, to renew a Driver License the stipulation is: "You cannot change your address during this transaction. You must have a completed form MV-619 (Eye Test Report) for this transaction. Read the requirements before you begin this transaction". To order a duplicate registration sticker for your car and get a receipt on-line, the stipulation is that (1) it replaces your damaged, lost or stolen registration items but not plates, (2) your duplicate will arrive by mail. Allow up to 2 weeks; most orders arrive in a week or less, (3) you should not order on-line if your address has changed, (4) if your registration is due to expire within 60 days, you may choose to renew now instead of ordering a duplicate and (5) you should be aware that you may be ticketed if your vehicle does not display a valid registration sticker. The quickest way to get your window sticker and registration receipt stub is to go to a local DMV office http://nyc.gov

\subsection{Norms can be violated}

The second property in the definition of normative multiagent systems is that norms can be violated. This is also seen as an important condition for the use of deontic logic in computer science: "Importantly, the norms allow for the possibility that actual behaviour may at times deviate from the ideal, i.e. that violations 
of obligations, or of agents rights, may occur", as observed by Jones and Carmo [9].

If norms cannot be violated then the norms are "regimented". For example, if there is a norm in access control that a service can only be accessed with some certificate, then this norm can be implemented in the system by ensuring that the service can only be accessed when the certificate is presented too. Regimented norms correspond to preventative control, in the sense that norm violations are prevented.

When norm violations are possible there is only detective control, in the sense that behavior must be monitored, and norm violations have to be detected and sanctioned. "social order requires social control, an incessant local (micro) activity of its units, aimed at restoring the regularities prescribed by norms. Thus, the agents attribute to the normative system, besides goals, also the ability to autonomously enforce the conformity of the agents to the norms, because a dynamic social order requires a continuous activity for ensuring that the normative systems goals are achieved. To achieve the normative goal the normative system forms the subgoals to consider as a violation the behavior not conform to it and to sanction violations" 7 .

Norms can be violated because they are soft constraints. In digital cities, disincentives are often the mechanism used to prevent users from infringing their norms. In point (5) of the previous example, if you don't have a valid registration sticker on your windshield you may be ticketed. Similarly, most digital cities offer online appointments for pick up of large objects or appliances containing Freon, such as refrigerators, by their sanitary department. They clearly stipulate that illegal dumping is forbidden and will be fined, in Paris 183 euros.

When norm violations are possible, there are normative multiagent systems in which the violations can trigger new obligations, the so-called contrary-to-duty obligations. With contrary-to-duty obligations, there is not only a distinction between ideal and bad behavior, but there is also a distinction between various degrees of sub-ideal behavior.

Norm violations can lead to sanctions for compensation, or attempts to undo the violation (roll-back in database systems). When there is a sanction, the repair action can either be completely undone (as for example in business or economics) or we can remain in sub-ideal state (as in some kind of moral reasoning).

\subsection{Where do they come from?}

The definition of normative multiagent systems does not say where norms come from. There are many ways in which norms can be created. For example, in digital cities "government regulations extend laws with specific guidance to corporate and public actions" [2].

\subsection{Can they change overtime?}

A third issue in the definition of normative multiagent systems is that agents can modify norms. 
In many electronic institutions the norms are fixed and cannot be changed within the system, but in many organizations there are roles defined within the system. The question is whether digital cities are a collection of electronic institutions, in other words, are manipulation and changes allowed within the system? In the US the site Regulations.gov provides a national forum for users to comment on existing and pending federal rules.

\section{Definition of Conviviality}

\subsection{Introduction}

The concept of conviviality often comes up in the context of web communities to describe sociable and forthcoming relations but it also arises in institutional contexts to generically denote the more specifically human qualities of communication: fun, easy going, friendly, cheerful, lively or polite. Looking at the term frequency on the Europe Information Society Thematic portal, if one enters today "convivial" in the search box, the result pages list 65 different documents. Indeed, in 1998 the European Community developed its strategy to promote conviviality as shared social value and selected it as research theme, one out of four, for its 5th Framework program for research (1998-2002): Societe de l'Information Conviviale, User-Friendly Information Society. It first called for large scale projects and programs that promoted user empowerment, human interactions, ambient intelligence and distributed services, for the elaboration of projects ranging from Content4All (2004-2006), to Humaine (2004-2008) to Companions (2006-2010) and programs such as the Convivio Net Consortium (2003-2005) developing convivial technologies, that is people-centered; All these initiatives seek to address the growing challenges in digital cities: need to support new interaction and communication paradigms, to bridge the increasing digital divides between social groups and remedy nascent social fragmentation and isolation by increasing social cohesion and community identity.

Generally speaking, a convivial place or group is one in which individuals are welcome and feel at ease [10] [11] [12, but definitions in literature spread from individual freedom realized in personal interdependence [13] to rational and cooperative behavior [14] to normative instrument when in the hands of power at play 15 .

In this section we raise the following question: Which definition of conviviality can be used and operationalized for digital cities? By means of information and communication technologies, digital cities are virtual presences and extensions of our physical cities. Started in 1990 (see Figure 1) they divide into five broad categories [16]: Non-profit grass-root community initiatives from the early days, municipal information and communication networks now referred to as e-governments, commercial city-oriented web sites such as AOL Digital Cities and MSN Citisearch, virtual environments for virtual communities for example communities of interest and finally information and communication technology (ICT) experiments. Although initially an American phenomena, the European 
Community quickly caught up in '93 with Telecities Network and in 2000 a 30year plan encouraging member countries to build their own digital cities baed on common vision. Each step of the plan corresponds to a technological level: the current stage focuses on establishing systems interoperability, the following one on Intelligent City Systems (2009), then Ambient Intelligence (2013) and finally Smart Cities (2030). The principal objectives are to "transform and modernize local administrations in order to improve the level and quality of life of the population at both individual and community levels" [16], for example providing $24 / 7$ access to services and content to reduce waiting lines and traffic congestion, or multilingual functionalities to reflect the linguistic diversity, facilitate inclusiveness process and reinforce social cohesion.

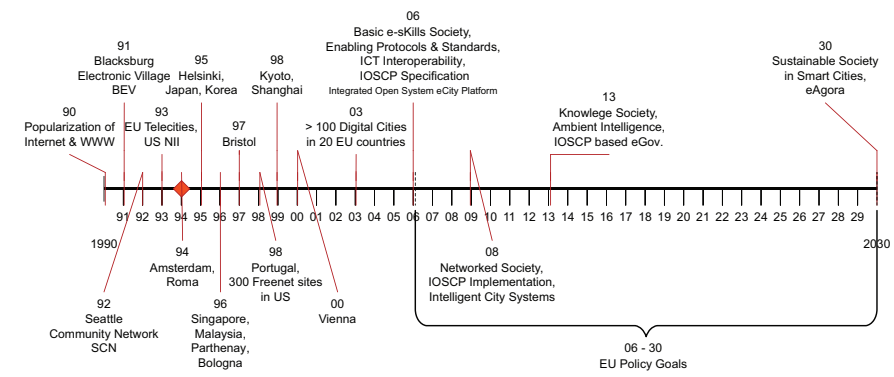

Fig. 1. Timeline: Conviviality and Ambient Intelligence

The question breaks down into the following questions: what kinds of notions of conviviality exist and how can these notions be used for digital cities. The methodology we follow for this section is a literature review in the areas of semiotics, philosophy, sociology, computer science, agent theory and human computer interaction. We then proceed with critical discussions. Our success criteria are the generality of our model and the evaluation and measure of conviviality. As stated by Sadek et al. [14], conviviality is "the essential and global characteristic of services () it emerges from the intelligence of the system and not from a set of local characteristics". Indeed, the local characteristics or criteria that determine conviviality "vary depending upon the application context and the types of users" [14]; consequently a list of criteria will not add up to a conviviality value. The critical factors are on one side the relation that binds the criteria together and on the other side, the way this relation is perceived by individuals. Furthermore, criteria are defined for specific contexts: security for banks, trust for relationships, flexibility and adaptability for web interface, scalability, efficiency and speed for systems, user density for given locations, group stability for communities of interest and so on.

In this paper we do not review the aspects of belief, desire and intentions of agent theory. Also out of scope are game theoretical approaches, the notion of equilibrium inherent to the temporal dimension of group behaviors and cost- 
benefits analysis from economics theory. The layout of this section is as follows. In each section we first give an overview of the kinds of notions of conviviality existing in the field and then discuss how these notions can be used in digital cities. We start with socio-cognitive approaches, then we consider computer science, agent theory and multi-agent systems, and finally Human Computer Interaction.

\subsection{Socio-Cognitive Approaches}

The role of conviviality in social systems Conviviality describes a relation not only between the individuals of a group but also between groups. As power shifts between groups so does the way groups and individuals relate to each other. Conviviality then addresses the relations between groups of different characteristics, minority and majority groups and consequently the concepts of exclusion, the outsiders being kept away, and intrusion, the intruders forcing their way into the excluding group. This process reveals the dynamic aspect of conviviality and its temporal dimension and raise the questions of how is conviviality created, how it evolves and what makes it fail.

Definitions of conviviality

Looking at some definitions shows that the meaning of conviviality depends from the context of use (table 1). For example, a convivial technology applies to the relation human-computer and refers to how easy, efficient and intuitive it is for a human to use this technology; whereas in sociology it applies to the relation human-human and refers to the equally beneficial quality of the relation.

Table 1. Definitions of conviviality

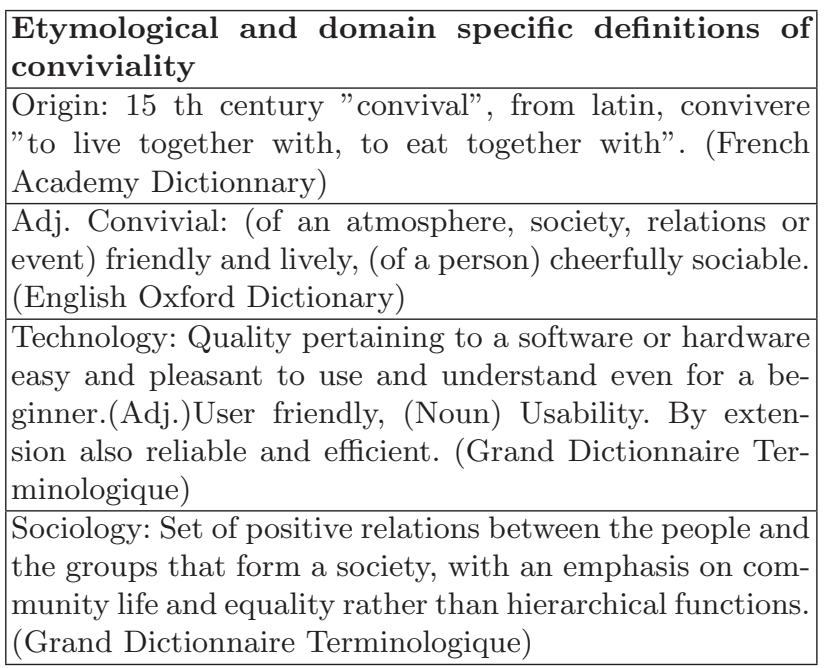


A less common view of conviviality is when it becomes an instrument to exercise power and enforce one point of view over another. Conviviality is then experienced as a negative force by the loosing side. We summarized from different sources the positive and negative aspects of conviviality and as example present excerpts (table 2). Clearly, the positive aspects of conviviality emphasize the sharing of common grounds whereas the negative aspects emphasize division and coercive behaviors.

Table 2. Conviviality: Positive and negative aspects

\begin{tabular}{|c|}
\hline Positive aspects (enabler) \\
\hline Share knowledge and skills \\
Deal with conflict \\
Inclusiveness \\
Equality \\
Trust \\
\hline Negative aspects (threat) \\
\hline Crush outsiders \\
Fragmentation \\
Totalitarism \\
Reductionism \\
Deception \\
\hline
\end{tabular}

Individuals vs. groups

Being the first in 1958 to use conviviality in a scientific and philosophical context, Polanyi [17] describes it as synonymous with empathy "which alone can establish knowledge of other minds". Through empathy individuals identify with others, it provides a way to understand other individuals by experiencing their feelings, thoughts and attitudes thereby acquiring personal knowledge, yours and theirs. Polanyi further describes in 1974 [18] a community as convivial when it aims at sharing knowledge: members trust each others, share commitments and interests and make mutual efforts to create and preserve conviviality.

In his 1971 critical discourse on education, Deschooling Society [19], Illich defines a convivial learning experience as one based on role swapping: teacher role alternates with learner role and vice versa, thereby emphasizing the concept of reciprocity as key component to conviviality. It is however with Illich's 1973 publication of Tools for Conviviality [13] that the concept really acquires a new dimension as it is defined as "an intrinsic ethical value". For Illich, conviviality is synonymous with "individual freedom realized in personal interdependence", it is the foundation of a new society one that gives its members the means, referred to as tools, for achieving their personal goals: "A convivial society would be the result of social arrangements that guarantee for each member the most ample and free access to the tools of the community and limit this freedom only in favor of another member's equal freedom.". 
However it is Putman and his colleagues who in the 80's take the concept of conviviality further as an enhancement to social capital and in 1988 refer to conviviality as a "condition for civil society" [20. Putnam later argued then later in 2000 that in a civil society "communities are characterized by political equality, civic engagement, solidarity, trust, tolerance and strong associative life" [21]. These are the values found today in e-governments charters that aim at increasing social cohesion and inclusiveness, by putting citizens at the centre of technological change.

In 2004 Schechter, taking part to a semiotics symposium on conviviality, takes another look at the concept adding that "in a basic sense, conviviality is a social form of human interaction" [12. She binds interaction to experience and recognizes the social dimension of conviviality as a way to reinforce group cohesion through the recognition of common values. "Thus the sharing of a certain kind of food and/or drink can be seen as a way to create and reinforce a societal group through a positive feeling of togetherness (being included in/or part of the group), on which the community's awareness of its identity is based." Schechter transforms the physical experience of conviviality into a learning and knowledge sharing experience. "To know is to understand in a certain manner that can be shared by others who form with you a community of understanding".

From groups to institutions

One cannot impose conviviality to a group claims Lomosits, it is a "consultative process that can only be recommended" [22] and reached through a consensus.

However, Hofkirchner explains that it is the "normative idea of unity-throughdiversity that deserves attention when applying conviviality to the level of world society" 23. The author then examines the unity-diversity relation. He first replaces the terms unity-diversity with identity-difference and then describes the four resulting scenarios: (1) "establish identity by eliminating difference at the cost of the differentiated side" yielding reductionism and universalism or (2) "of the undifferentiated side yielding unity without diversity", that is particularism, totalitarism and homogenization; (3) "establish difference by eliminating identity yielding diversity without unity", that is fragmentation and (4) "establish identity in line with difference yielding unity and diversity". The achievement of conviviality is in this integration of difference and differentiation of identity. Among other examples, it yields transculturalism.

Somov precises the normative aspect of conviviality in that it belongs to the regulation of human interrelations: "Conviviality (just like conflicts) is based on agreements or contradictions" [24].

According to Lamizet, conviviality was elaborated to describe both "institutional structures that facilitate social relations and technological processes that are easy to control and pleasurable to use" [25]. Conviviality emphasizes individual expression facilitated by personalized interface and customized content, while it also contributes to media standardization and the uniformization of representation systems.

The darker side of conviviality 
"Conviviality is achieved for the majority, but only through a process by which non-conviviality is reinforced for the minority" states Ashby 26]. This aspect of conviviality rarely considered brings negative results along with the expected positive ones. Ashby further reveals the instrumentalization of conviviality to favor one group at the expense of another: "truth realities about minorities are built from the perspective of the majority via template token instances in which conflict is highlighted and resolution is achieved through minority assimilation to majority norms" [26].

Taylor Taylor [15] goes further: "Conviviality masks the power relationships and social structures that govern communities." The author explores the contradiction between institutions and conviviality raising the question "whether it is possible for convivial institutions to exist, other than by simply creating another set of power relationships and social orders that, during the moment of involvement, appear to allow free rein to individual expression". In Taylor's view community members "may experience a sense of conviviality which is deceptive and which disappears as soon as the members return to the alienation of their fragmented lives."

The use of conviviality for digital cities

Users go to city web portals to fulfill needs for administration documents, official information, entertaintment and so on. When trying to accomplish their tasks, users have to struggle with a number of constraints and must continuously compromise. Constraints come from other users competing for resources or imposing their behaviors, from city administrations information and identity controls, or technical reasons such as system overloads or lack of functionality. Groupware, communityware and other research areas explore collaborative tools and systems however, there is to date no formal model for integrating notions like conviviality.

Conviviality affects coalition formation among humans by motivating individuals to associate with each other: it allows more efficient learning and reinforces social cohesion. Conviviality affects knowledge sharing and encourages cooperative behaviors, both constitutive values for digital cities. Moreover, conviviality contributes to reducing mis-coordinations that result from breakdowns in shared knowledge.

Some open questions are for example how to avoid reducing conviviality to one of its components and how to preserve its core value and meaning? A problem with formalization and implementation is that the concept of conviviality itself is inherently non-formal, when for instance you formalize it, the result may be a set of rules, norms, notions such as trust, reputation, bonus points, or other economic notions that miss the point. On analysis, conviviality may disappear and be reduced to other notions.

\subsection{Computer Science Approaches}

The role of conviviality in Multi-agent systems (MAS)

In multi-agent systems an agent is defined as "a computer system that is situated in some environment, and that is capable of autonomous action in this 
environment in order to meet its design objectivesAgents are capable of flexible (reactive, proactive, social) behavior" 27. This capability is crucial since it allows agents to cooperate, coordinate their actions and negotiate with each other.

The use of conviviality for Intelligent Tutoring Systems

The system proposed by Gomes et al. 28 provides a recommendation service of student tutors for computational learning environments. "Each agent pupil represents a pupil logged onto the system. One of the functions of the system is to be the client for an instant message service. Through its agent pupil, any pupil can communicate with other pupils in the system". Another function of agent pupils is to pass information, inferred by the agent or adjusted by pupils, on the pupils' affective states.

The authors' claim that "convivial social relationships are based on mutual acceptance through interaction" hence on reciprocity, here students helping each other. A utility function takes as input students' social profiles, computes students' affective states indicating their need of help and then recommends tutors. Remaining challenges are to define inputs for utility functions computing recommendations, presently random values, and automated inference of students in need. These critical tasks show the importance of further research in evaluation methods and measures for concepts such as mood, sociability and conviviality.

The use of conviviality for Conversational Agent

"All service offerings must integrate conviviality to the interaction between user and system as an essential preoccupation" [14]. To fulfill this goal, Sadek et al. define a convivial agent as rational and cooperative, consequently an interaction is convivial "if the agent presents, jointly and at all times, one or all of the following characteristics: Capacity for negotiation, contextual interpretation, flexibility of the entry language, flexibility of interaction, production of co-operative reactions and finally of adequate response forms." These communicative capacities and social intelligence based on emotional intelligence are crucial to enhance agents' ability to interact with users.

Building on this work Ochs et al. 29] distinguish felt emotions from expressed emotions noting that "a person may decide to express an emotion different from the one she actually felt because she has to follow some socio-cultural norms". We believe this direction to be very relevant to the evaluation of conviviality as it dissociates personal from social expression.

The use of conviviality for reputation systems

Reputation is defined as "the overall quality or character as seen or judged by people in general and the recognition by other people of some characteristic or ability" [30. When Casare and Sichman state that "reputation is an indispensable condition for the social conviviality in human societies" [31] they emphasize this overall quality that both reputation and conviviality share. The authors' system insures that everyone is aware of anyone that complies or not to the rules of the group. The authors define a functional ontology of reputation for multi-agent systems whereby "roles are played by entities involved in reputative processes such as reputation evaluation and reputation propagation." 
The authors' claim that "concepts of the legal world can be used to model the social world, through the extension of the concept of legal rule to social norm and the internalization of social mechanisms in the agent's mind, so far externalized in legal institutions". The agents actual behaviors are therefore compared to the social norms observed in their world. The process however presupposes an initial reputation profile of users that agents can then update in real time. Reputation acts as a communication tool ensuring complete social transparence throughout the system. The strict application of norms to reputation however may be difficult and suffer from rigidity. Of course, the same holds for conviviality.

The role of norms in multi-agents systems and how it applies to conviviality

The role of norms is increasingly getting attention specifically in multi-agents systems (MAS) where the most common view is that "norms are constraints on behavior via social laws" 4. Boella et al. describe action models where "agents are goal directed and try to maximize their choice of means to obtain a goal". Moreover, it is assumed that an agent belongs to a group and must follow the norms like all members of that group. Similarly, using conviviality for a digital community maximizes the community benefits, for instance by reducing conflicts during communications allowing efficient interactions and cooperation. Indeed, conviviality, like politeness, uses the group rules to regulate members' interactions.

Boella et al. enunciate the three different functions of norms as follows: "we have norms that are of a constitutive nature, they define the agent's membership in a system of action, and the system of action at large. Another function of norms is regulation, describing what members of a social system must and must not do. Thirdly, norms may have a distributive function that is how rewards, costs and risks are to be divided among the social system's members" 4]. Similarly, conviviality reinforces social cohesion by reflecting its core values internally as well as externally. It also contributes to optimize performances within communities as well as between communities, improving coordination in the city. Finally, conviviality is enforced through mechanisms such as the expression of feelings: praise and encouragements for members who conform to the rules, anger and blame for the ones who do not.

\subsection{Human Computer Interaction (HCI) Approaches}

\section{Ambient spaces and playthings}

In her study of animated toys, Ackermann, looking at the relational qualities of playthings notes that beyond humanoid traits, it is an AniMate's manners of interaction that matter: "Beyond smarts, it is its conviviality. Beyond obedience or bossiness, it is an AniMate's relative autonomy and ability to share control" [10. Ackermann emphasizes the exploration of partial and shared control as critical quality of conviviality. Merging digital and physical elements, she now experiments with ambient places, Piazzas, defined as convivial spaces and that function as transitional zones or third-space, between work and home.

Pedagogy 
According to Sipitakiat, conviviality establishes a "dynamic equilibrium in the interplay between different modes of learning and teaching" [11]. In his research, based on constructionism and on Illich theories, the author asserts that "people learn with particular effectiveness when they are engaged in constructing personally meaningful artifacts (such as computer programs, animations, or robots)." The author stresses that conviviality encourages people to produce information and content rather than just consume it.

\section{User-friendly vs. convivial}

In HCI until recently, the terms user-friendly and convivial were often synonymous. However, the distinction increased as factors such as emotional experience and enjoyment were taken into consideration, user-friendliness referring now more to qualities such as ease-of-use, compliance to ergonomics standards and usability rules whereas the notion of conviviality finds new meaning with HCI developments in areas such as adaptive systems, augmented cognition, multi-modal interactions and ambient intelligence. Ackerman's research trying to define the qualities that make convivial spaces is just one of many examples relevant to the user of conviviality for digital cities.

\subsection{Other Related Works}

\section{Artificial sociable companions}

The Companions that Wilks envisions 32 ] are permanent software agents attached to single users. They act as intermediaries for all information sources that users cannot manage. For instance, Companions for seniors provide company to senior citizens who feel lonely, they act as technical task assistant to search the web for travels or keep track of events their owners forget. Conversely, Companions for juniors provide assistance with teaching, explanations-on-demand and advices.

Mixed-Initiative Interaction In a rather new area of research called mixed-initiative interaction "people and computers take initiatives to contribute to solving a problem, achieving a goal, or coming to a joint understanding" [33. A critical element is how users focus their attention: "Attentional cues are central in decisions about when to initiate or to make an effective contribution to a conversation or project" 34. Mixed-initiative research aims at developing software that filters appropriately incoming information to shield users from incoming disturbances such as emails and phone calls. The filtering of incoming information is achieved through measuring user's keystrokes and scrolling activities, recording the number of opened windows, analyzing content, checking events in calendars, location and time of day and so on.

The cognitive dimension of conviviality Research on sociable companions, information filtering, interruption and distraction clearly exemplifies the cognitive aspects of conviviality. It also suggests wide ranging uses for digital cities: from individual social assistant, to group communications, to regulation of emergent behaviors. 


\subsection{Our definition}

We summarize by first noting that conviviality is usually considered a positive concept but that a darker side emerges when it becomes the instrument of power relations. Secondly, to the question we raised: Which definition of conviviality can be used and operationalized for digital cities? We answer with this two-fold definition of conviviality as

1. a condition for social interaction and

2. an instrument for the internal regulation of social systems.

Consequently, we see the most important uses of conviviality in digital cities as a mechanism to reinforce social cohesion as well as a tool to reduce miscoordinations between individuals.

\section{Use of Norms for Conviviality}

In this section we reconsider the issues discussed in the context of legal and institutional norms for digital cities in the context of norms for conviviality.

\subsection{Kinds of norms}

The distinction between prohibitions and permissions (or rights) also occur for conviviality, as there are positive and negative aspects of this social concept.

Counts-as rules can be used to define kinds of conviviality.

\subsection{Explicit vs. implicit representation of conviviality}

Since most norms for conviviality are social norms, they are often not made explicit. Consider for example norms of being politically correct. Agents may appear to follow and embrace the beliefs of a group by fear of appearing different but without conviction, following a group without truly being part of it, and so on. Other implicit norms may refer to deception, non-transparent systematic controls, or to hide conflicts.

However, some explicit norms are relevant for conviviality. Explicit norms relevant for conviviality can refer to cooperation among agents, for example the protocol of communication, For example, http was successful thanks to its "conviviality", its limitations made it "popular" since they enhanced its ease of implementation. Ftp is successful too, for it compensates some of the http limitations, and both ftp and http are very cooperative. As another example, the digital city Issy les moulinaux [http://www.issy.com/] put all its accounting books online to all citizens. 


\subsection{Conviviality can be violated}

There are many examples of violating conviviality. Ignoring cultural and social diversity is violating conviviality as it creates conviviality for a group at the expense of others. Another possible violation is being ignored when coming to ask advices to a city administrator. For example, the online Paris library assures members of a kind and pleasant service and proposes a free mediator service in case of difficulties dealing with city clerks. Yet another violation is to promote homogenization and enforce exclusion, or to crush outsiders, fragmentation, totalitarism, reductionism, deception and so on.

There are many possible solutions to conviviality violation, dealing with issues such as sharing knowledge and skills, deal with conflict, feeling of "togetherness", equality, trust and so on.

\subsection{Do norms change over time?}

Conviviality meant nothing to the IBM system developers in the 60's until the 90's. The IBM system command language "JCL" was deprived of any logic, coherence. Similarly the first IBM PCs did not even try to be accessible to the common users. Conviviality implied a form of democratisation of the use of computers. Eventually IBM turned to UNIX and Microsoft, with Windows 95 rediscovered that conviviality could be a strong marketing argument. Conviviality is then seen as a call to the user intelligence (whether he/she was a computer specialist or a simple end user), adaptability, curiosity, via a clear and communicable - i.e. teachable - protocol, aimed at providing an extensive control of systems which could be quite complex.

\subsection{Where do norms come from?}

The Socratic conviviality scheme, identifies two kinds of partners in the protocol: pseudo-passive ones (e.g. a guide, a master, a service provider, i.e., a reactive agent, but not one who takes the initiative) and active ones (e.g. those who are seeking assistance). The protocol consists in stimulating the intelligence and curiosity of the active partner, by questions of the pseudo-passive one. I.e. the active partner is considered an intelligent and autonomous agent : this active agent feels therefore he/she is a respected individual.

For instance, a tutoring system, asks as first question "may I help you?" and try to extract from a user answer - one could consider it as a Google query possible tracks e.g. towards tutorials. Or that system could attempt to evaluate the user understanding level in some discipline, via a set of questions, and reading suggestions.

As a conversational agent, the Eliza system proved to be genuinely convivial, though extremely simple. It fostered the desire of its users to express and uncover themselves, by simply answering (often dumm but unexpected) questions on a terminal, as if the user was lying on a psychiatrist couch. 
The Socratic protocol requires an open system, a system which needs permanent adaptations, since that protocol admits it will never be able to respond to all the user expectations, and the protocol has to have a "user criticism" or a "failure reports" section where users may report their frustration. The analysis of these user reactions is one of the major basis for the system enhancement and evolution.

Such a protocol could be quite "normative". This corresponds to the " conviviality" of an institutional relation, with its assumed teleology.

\section{Conclusion}

In this paper we consider contrast norms for conviviality with legal and institutional norms in digital cities. We consider the following issues. First, the kinds of norms typically distinguished in legal systems can be distinguished for norms of conviviality too. Second, norms for conviviality are often implicit, and we believe it is an important question when such norms should be made explicit. Third, the issue of violation of conviviality and ways to deal with it is of central concern in web communities like digital cities. Fourth, norms concerning conviviality should be able to change over time. Fifth, norms for conviviality can come from a wide variety of sources.

\section{References}

1. Caire, P.: A critical discussion on the use of the notion of conviviality for digital cities. In: Proceedings of Web Communities 2007. (2007)

2. Lau, G.T., Law, K.H., Wiederhold, G.: Analyzing government regulations using structural and domain information. IEEE Computer 38 (2005) 70-76

3. den Besselaar, P.V., Melis, I., Beckers, D.: Digital cities: Organization, content, and use. In: Digital Cities. (2000) 18-32

4. Boella, G., van der Torre, L., Verhagen, H.: Introduction to normative multiagent systems. Computational \& Mathematical Organization Theory 12 (2006) 71-79

5. Boella, G., van der Torre, L.W.N.: Regulative and constitutive norms in normative multiagent systems. In: KR. (2004) 255-266

6. Lawrence, D.G.: Procedural norms and tolerance: A reassessment. The American Political Science Review (1976)

7. Boella, G., van der Torre, L.W.N.: Constitutive norms in the design of normative multiagent systems. In: CLIMA VI. (2005) 303-319

8. Searle, J.R.: Speech Acts: An Essay in the Philosophy of Language. Cambridge University Press (1970)

9. Jones, A., Carmo, J. Handbook of Philosophical Logic. In: Deontic logic and contrary-to-duties. Kluwer Academic Publishers (2002) 265-344

10. Ackermann, E.K.: Playthings that do things: a young kid's "incredibles"! In: IDC '05: Proceeding of the 2005 conference on Interaction design and children, New York, NY, USA, ACM Press (2005) 1-8

11. Sipitakiat, A.: Digital technology for conviviality: Making the most of students' energy and imagination in learning environments. Master's thesis, MIT, Cambridge, MA,USA (2001) 
12. Schechter, M.: Conviviality, gender and love stories: Plato's symposium and isak dinesen's (k. blixen's) babette's feast. Trans, Internet journal for cultural sciences 1 (2004)

13. Illich, I.: Tools for Conviviality. Marion Boyars Publishers (1974)

14. Sadek, M.D., Bretier, P., Panaget, E.: ARTIMIS: Natural dialogue meets rational agency. In: IJCAI (2). (1997) 1030-1035

15. Taylor, M.: Oh no it isn't: Audience participation and community identity. Trans, Internet journal for cultural sciences 1 (2004)

16. Ishida, T.: Understanding digital cities. In: Digital Cities. (2000) 7-17

17. Polanyi, M.: Personal Knowledge: Towards a Post-Critical Philosophy. Routledge \& Kegan Paul Ltd, London (1958)

18. Polanyi, M.: Personal Knowledge : Towards a Post-Critical Philosophy. University Of Chicago Press (1974)

19. Illich, I.: Deschooling Society. Marion Boyars Publishers, Ltd. (1971)

20. Putnam, R.D.: Diplomacy and domestic politics: The logic of two-level games. International Organization 42 (1988) 427-460

21. Putnam, R.D.: Bowling alone: the collapse and revival of american community. In: CSCW. (2000) 357

22. Lomosits, H.: Future is not a tense. Trans, Internet journal for cultural sciences 1 (2004)

23. Hofkirchner, W.: Unity through diversity.dialectics - systems thinking - semiotics. Trans, Internet journal for cultural sciences 1 (2004)

24. Somov, G.Y.: Conviviality problems in the structure of semiotic objects. Trans, Internet journal for cultural sciences 1 (2004)

25. Lamizet, B.: Culture - commonness of the common? Trans, Internet journal for cultural sciences 1 (2004)

26. Ashby, W.: Unmasking narrative: A semiotic perspective on the conviviality/nonconviviality dichotomy in storytelling about the german other. Trans, Internet journal for cultural sciences $\mathbf{1}$ (2004)

27. Wooldridge, M.: An introduction to multi-agent systems. J. Artificial Societies and Social Simulation 7 (2004)

28. (Gomes, E.R., Boff, E., Vicari, R.M.)

29. Ochs, M., Niewiadomski, R., Pelachaud, C., Sadek, D.: Intelligent expressions of emotions. In: ACII. (2005) 707-714

30. Merriam-Webster, I.: Merriam Webster OnLine Dictionary. Merriam-Webster (2006)

31. Casare, S., Sichman, J.: Towards a functional ontology of reputation. In: AAMAS '05: Proceedings of the fourth international joint conference on Autonomous agents and multiagent systems, New York, NY, USA, ACM Press (2005) 505-511

32. Wilks, Y.: Artificial companions. In: MLMI. (2004) 36-45

33. Horvitz, E., Koch, P., Apacible, J.: Busybody: creating and fielding personalized models of the cost of interruption. In: CSCW. (2004) 507-510

34. Horvitz, E., Kadie, C.M., Paek, T., Hovel, D.: Models of attention in computing and communication: from principles to applications. Commun. ACM 46 (2003) $52-59$ 